\title{
Mario Gecchele, Paola Dal Toso (éd.), Educazione democratica per una pace giusta
}

Rome, Armando editore, 2010, 191 p.

\section{Michel Ostenc}

\section{(2) OpenEdition}

\section{Journals}

Édition électronique

URL : http://journals.openedition.org/assr/23969

DOI : $10.4000 /$ assr.23969

ISSN : $1777-5825$

Éditeur

Éditions de l'EHESS

Édition imprimée

Date de publication : 30 décembre 2012

Pagination : 186

ISSN : 0335-5985

Référence électronique

Michel Ostenc, «Mario Gecchele, Paola Dal Toso (éd.), Educazione democratica per una pace giusta » Archives de sciences sociales des religions [En ligne], 160 | octobre-décembre 2012, mis en ligne le 13 mars 2013, consulté le 21 septembre 2020. URL : http://journals.openedition.org/assr/23969 ; DOI : https://doi.org/10.4000/assr.23969

Ce document a été généré automatiquement le 21 septembre 2020.

(c) Archives de sciences sociales des religions 


\section{Mario Gecchele, Paola Dal Toso (éd.), Educazione democratica per una pace giusta}

Rome, Armando editore, 2010, 191 p.

Michel Ostenc

\section{RÉFÉRENCE}

Mario Gecchele, Paola Dal Toso (éd.), Educazione democratica per una pace giusta, Rome, Armando editore, 2010, 191 p. 
1 Cet ouvrage collectif est un hommage rendu à l'action militante et à l'œuvre éducative du doyen de la Faculté des Sciences de la Formation de l'université de Vérone. Emilio Butturini a appartenu dans les années 1960 au Mouvement étudiant d'Action catholique en Italie. Il a été membre pendant des décennies du Conseil central de l'Union catholique italienne des Enseignants du secondaire et siégé au Conseil municipal de Vérone de 1994 à 2002.

2 La Constitution de la République italienne avait reconnu les Accords du Latran et l'enseignement de la religion restait obligatoire dans les écoles publiques. La lettre des évêques du 25 mars 1960 dénonçait encore le laïcisme comme le

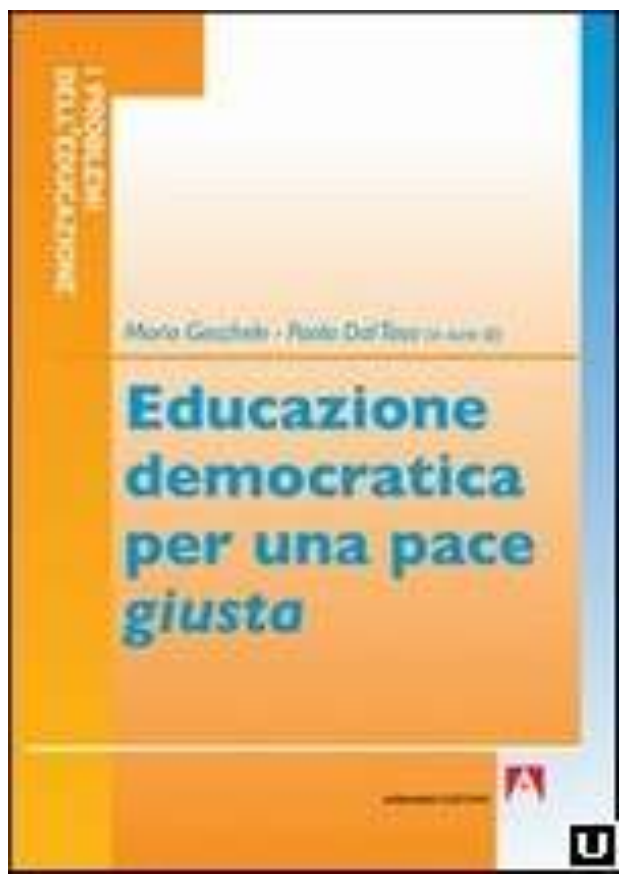
principe inspirateur des erreurs morales des temps modernes. Le concile Vatican II marqua un tournant historique de l'époque post-tridentine et ses décrets sur la liberté religieuse montrèrent à beaucoup de catholiques italiens combien le concordat de 1929 était inadapté à leur époque. La « Lettera a una professoressa » (1967) de Lorenzo Milani, que Butturini considérait comme un maître de liberté et de responsabilité, eut un profond retentissement dans le mouvement catholique étudiant italien. Elle préconisait une école publique libérée de tout préjugé idéologique ou religieux. Emilio Butturini fut profondément imprégné du lien personnaliste chrétien entre éducation et démocratie. L'«Humanisme intégral » de Jacques Maritain en était la charte fondamentale qui trouvait son application dans " L'éducation à la croisée des chemins " (Giancarlo Galeazzi, Il problema della scuola pubblica in Francia, Brescia, 1976). Butturini appréciait en outre chez Emmanuel Mounier la passion du croyant qui n'excluait aucune liberté d'esprit; mais son engagement puisait ses origines dans la mission sociale de l'Église. Il avait lui-même suivi les enseignements des écoles fondées à Vérone par Nicola Mazza où la motivation des élèves et la compétence des maîtres contribuaient à la formation d'authentiques communautés éducatives (Emilio Butturini, Rigore e libertà. La proposta educativa di don Nicola Mazza, Vérone, 1990). Il ne cachait pas non plus son admiration pour Gaspare Bertoni, le fondateur des "écoles de charité » de Vérone (Emilio Butturini, Don Gaspare Bertoni al centro d'una mirabile fioritura di santità, in : S. Gaspare Bertoni, angelo del consiglio, Vérone, 2004 , p. 201-213). Butturini fut également marqué par la pédagogie de Giovanni Calabria qui prônait les vertus de l'exemplarité dans le respect de la personnalité de l'enfant (Emilio Butturini, Contesto storico con particolare attenzione alla realtà veronese, in : Storia dell'Opera Don Calabria. Il contesto storico e le case di San Zeno in Monte, Costozza ed Este, t. 1, Vérone, 2007, p. 19-94). L'intérêt de Butturini pour ces expériences éducatives s'élargira plus tard à l'ensemble des établissements scolaires de Vérone (Emilio Butturini, Istituzioni educative a Verona tra '800 e '900, Vérone, 2001 ; Emilio Butturini, Istituzioni educative veronesi tra fine Ottocento e primo Novecento e Movimento sociale 
cattolico, in : M. Ferrari (dir.), Davide De Massari. L'azione pastorale e sociale tra Annessione e Grande Guerra, Vérone, 2006, p. 55-74).

3 Les mouvements étudiants de 1968 n'avaient pas entamé la conviction de Butturini qui voyait dans l'école un lieu capable de concilier la démocratie représentative avec une démocratie participative qui avait sa sympathie (Emilio Butturini, L'esperienza di Verona, in: A. Agazzi (dir.), Professioni e studenti in una scuola rinnovata, Rome, 1969). Cet engagement militant l'incita à un retour aux sources de la pensée démocratechrétienne, en montrant tout ce qui l'opposait à la philosophie idéaliste et au fascisme concordataire. Certes, les catholiques avaient reconnu à la réforme scolaire de Giovanni Gentile (1923) le mérite d'avoir introduit un enseignement obligatoire de la religion à l'école primaire, mais ils lui reprochaient une conception qui en faisait une philosophie mineure. Le Parti Populaire de Don Sturzo préconisait au contraire un renouveau de l'école publique qui passait par une décentralisation capable d'assurer l'autonomie des établissements scolaires et la participation des familles à leur gestion (Emilio Butturini, La riforma Gentile e $i$ cattolici italiani, « Humanitas ", $n^{\circ}$ II, novembre 1975, p. 975-1008). Une conception de l'école comme communauté éducative s'opposait aux structures autoritaires fascistes et elle incitera plus tard Butturini à faire l'éloge de l'action politique du ministre de l'Instruction publique Guido Gonella, de la Constituante aux gouvernements d'Alcide De Gasperi (Emilio Butturini, Guido Gonella : dagli " Acta diurna » alla Costituente e al governo, nel segno della cultura antifascista, in : La memoria per il futuro, Vérone, 1996, p. 19-54).

4 La réforme de l'école maternelle de 1969 «Orientamenti dell'attività educativa nelle scuole materne statali » du ministre démocrate-chrétien Mario Ferrari Aggradi clôturait un long débat parlementaire. Elle insistait sur le caractère aconfessionnel d'une éducation religieuse destinée à développer des rapports fraternels entre les enfants. Cette réforme était de nature à faire naître un pluralisme et sa valeur humaine fut saluée par beaucoup de catholiques. Certes, le concile avait insisté sur le droit des parents à exiger que l'enseignement dispensé à leurs enfants ne heurtât point leurs convictions religieuses; mais il ne faisait pas de distinction entre l'enseignement de la religion et celui de la culture religieuse. Butturini estimait que la réforme allait dans le bon sens et il préconisera plus tard l'abandon du catéchisme dans l'école publique au profit d'une culture religieuse promue par l'État (Emilo Butturini, La religione a scuola. Dall'unità d'Italia a ogg, Brescia, 1987).

5 Les années 1970 virent d'importantes initiatives de l'État italien en matière scolaire. Le cycle didactique proposé par le ministre Ricardo Misasi au début de l'enseignement secondaire se heurta à l'opposition du parlement (Ricardo Misasi, Questa scuola impossibile, Rome, 1972). Le référendum sur le divorce de mai 1974 révéla l'existence d'une société italienne plus laïcisée que prévu et les catholiques se divisèrent sur l'opportunité d'en déduire une révision du système concordataire. Le ministre Franco Maria Malfatti suggéra une réforme de l'ensemble du secondaire avec une première année d'orientation, trois années d'enseignement et une cinquième d'approfondissement de l'orientation choisie. Les études comportaient des matières communes, des disciplines d'orientation et d'autres à option. Le projet impliquait la prolongation à quinze ans de l'obligation scolaire et il portait à dix-huit ans l'âge de la fin de l'enseignement secondaire; mais cette réforme s'enlisa elle aussi dans les navettes parlementaires entre la Chambre et le Sénat. Pendant que le grand dessein d'une loi organique perdait de sa crédibilité, on s'attachait à des mesures concernant la 
formation professionnelle (Giorgio Chiosso, Cultura, lavoro e professione, Milan, 1981), à la participation des parents et des élèves au fonctionnement des établissements scolaires. Butturini espérait que la présence d'étudiants dans les conseils universitaires ramènerait un esprit de tolérance dans l'enseignement supérieur (Emilio Butturini, La partecipazione degli studenti alla gestione della scuola : bilancio degli atteggiamenti dei giovani di fronte ai decreti delegati, in : G. Milanesi (dir.), Educazione e Politica. Società e politica nella scuola italiana, Turin, 1976, p. 207-226). La loi du 30 juillet 1977 et ses décrets d'application entendaient donner une réponse institutionnelle aux mouvements étudiants. Une école enfermée jusque-là dans une conception exclusive de transmission des connaissances s'ouvrait à l'idée d'une communauté éducative, mais les étudiants n'étaient pas encore considérés comme des sujets autonomes et ils se désintéressèrent vite du fonctionnement des nouvelles institutions. La loi du 16 juin 1977 supprimait les disciplines facultatives et imposait l'obligation d'une éducation musicale et technique. Elle regroupait l'enseignement des sciences mathématiques, physiques, chimiques et naturelles, en y incorporant l'éducation sanitaire. Elle renforçait enfin l'enseignement linguistique en y ajoutant des références aux origines latines de la langue italienne. Le ministre Malfatti nomma une "commission des soixante" présidée par le sénateur Fassino pour préparer les nouveaux programmes, et celle-ci préconisa la création d'un enseignement d'éducation religieuse qui suscita de vives polémiques, cependant Butturini considérait comme un progrès le fait que cette éducation fût une des finalités de l'école.

6 L'absence de réforme fondamentale donna lieu à de vifs débats. Les décisions prises apparaissaient comme des palliatifs correspondant à des principes généraux plus qu'à des critères concrets (Fabrizio Ravaglioli, Un riformismo alla deriva. Educazione e scuola degli anni '70, Rome, 1983; Giorgio Chiosso, Pedagogia e scuola in Italia tra utopia e riformismo (1968-1983), in: S. Serena Macchietti, Questioni di storia della scuola italiana (1945-1985), Quaderni dell'Istituto di Pedagogia dell'università di Siena, 1986). La plupart des commentateurs imputaient ces faiblesses à une séparation des domaines sociaux et culturels que l'on maintenait pour préserver leur spécificité et leur efficacité. Ces barrières étaient jugées responsables des aspirations révolutionnaires de la jeunesse. Butturini présentait l'école comme un fait structurel au service du système économique et social plus que de la culture (Emilio Butturini, Giovani, droga e rapporto educativo. Esperienza di un corso di formazione per insegnanti, Vérone, 1979). Les jeunes percevaient l'école comme un appareil idéologique de l'État qui bloquait toute initiative spontanée. Certes, les réformes des années 1970 avaient mis en place de nouvelles structures libéralisant l'accès à l'université, démocratisant le fonctionnement des établissements scolaires et rajeunissant le personnel enseignant, mais elles n'avaient pas amélioré les capacités de formation et il en résultait une marginalisation des jeunes par rapport au monde du travail qui s'accompagnait de leur désaffection pour les institutions. Ils conservaient toutefois une vision positive de la politique conçue comme un engagement personnel et une gestion sociale du bien commun. Plusieurs ouvrages étudiaient le comportement des jeunes dans leur famille (L. Ricolfi, L. Sciolla, Senza padri né maestri, Bari, 1980 ; G. Calvi, Comportamenti e valori: interpretazione provvisoria del decennio: 1970-1980, in : Atti del convegno "Società italiana e coscienza giovanile verso gli anni "ottanta" ", Milan, 1980). Butturini se préoccupait du malaise ressenti par les jeunes dans leur famille (Emilio Butturini, Crisi del rapporto tra i giovani e le istituzioni, "Pedagogia e Vita », Série 45, décembre 1983-janvier 1984, p. 133-151). Il en décelait les origines dans une mutation qui rendait plus difficile les rapports entre les générations. 
Désormais, la transmission des valeurs ne se faisait plus d'une génération à l'autre, mais d'une jeunesse à l'autre. Les parents confrontés à ces difficultés répugnaient à un autoritarisme devenu incompatible avec l'acte d'éducation, tout en regrettant une permissivité synonyme d'une démission devant des choix indispensables. Butturini dirigea à Vérone en 1980 et 1981 un séminaire consacré au renoncement des parents dans l'éducation de leurs enfants, alors que ces derniers avaient besoin de repères et de références. Les travaux portèrent sur les activités proposées aux jeunes pendant leur temps libre (Emilio Butturini, dir., Per un impiego alternativo del tempo libro giovanile, Padoue, 1983). La pédagogie sociale de Butturini entendait remédier au malaise de la jeunesse par une riposte globale de la société au niveau des valeurs morales (Emilio Butturini, Disagio giovanile e impegno educativo, Brescia, 1984).

7 Les laïcs proposaient de remplacer l'enseignement de la religion par celui des « Droits humains ». Cette discipline nouvelle aurait été l'aboutissement de la «religion de la liberté » chère à l'historicisme de Benedetto Croce et s'inspirerait de la « religion des religions » que John Dewey concevait comme le supplément d'âme de la démocratie. Les catholiques souhaitaient promouvoir deux enseignements distincts, l'un de culture religieuse professée par des enseignants dépendant de l'autorité scolaire et l'autre de religion catholique dispensé par des maîtres soumis à l'autorité ecclésiastique, mais deux variantes venaient ensuite. La «double voie» rendait la culture religieuse obligatoire, l'autre enseignement restant facultatif. L'autre variante préconisait le choix entre les deux disciplines. Butturini était favorable à l'option obligatoire (Emilio Butturini, Lo statuto professionale dell'insegnante di religione: curricoli di qualificazione, criteri di idoneità, in: Società civile, scuola laica e insegnamento della religione, Brescia, 1983, p. 295-337). Les programmes scolaires de 1985 furent très critiqués. La religion, "fondement et couronnement » de l'enseignement concordataire, passait désormais après les disciplines sociales. Elle était en outre démantelée, le problème historique de la religion étant réservé à l'enseignement de l'histoire. Les laïques craignaient une multiplication d'enseignements présentant un caractère religieux comme le souhaitaient certains catholiques favorables à une présence religieuse diffuse dans les diverses disciplines. Cette solution soulevait les objections de Butturini, les enseignants des différentes matières n'ayant pas reçu de formation à la problématique religieuse. La majorité des catholiques s'inquiétaient par contre d'une réduction du contenu religieux de l'enseignement de la religion qui échappait au contrôle de l'Église. Butturini s'insurgeait contre des mesures susceptibles de priver les élèves de toute culture religieuse et il renouvelait l'avertissement lancé par Carlo Iemolo à propos des conséquences que la mutilation du fait religieux pouvait avoir sur l'unité de certains peuples. Le pédagogue de Vérone dénonçait enfin le caractère démagogique d'une disposition de janvier 1986 qui invoquait la liberté de conscience pour donner à des élèves âgés de treize à quatorze ans le droit de refuser de suivre l'enseignement de la religion. Le débat se poursuivit jusqu'à l'arrêt de la Cour constitutionnelle d'avril 1989 reconnaissant la laïcité comme principe supérieur de la République italienne. L'école publique devait favoriser l'étude du fait religieux, tout en évitant de le privilégier au détriment d'autres conceptions de la vie. Pour Butturini, l'enseignement de culture religieuse ouvert à tous devait comporter une étude historique des problèmes religieux et une approche œcuménique de la Bible ainsi que des textes sacrés (Emilio Butturini, Profilo storico dell'insegnamento di religione cattolica in Italia, in: Z. Trenti, dir., Manuale dell'insegnante di religione. Competenza e professionalità, Turin, 2004, p. 13-28). 
8 L'intérêt du pédagogue de Vérone pour un enseignement de culture religieuse était révélateur de préoccupations profondes dépassant largement le credo d'un catéchisme. Une éducation prenant en compte la tradition chrétienne était capable de renouer avec le message évangélique pour résoudre le problème toujours actuel de la paix, élément fondamental de l'histoire de l'Occident (Emilio Butturini, L'educazione alla pace nella scuola, in : L. Corradini, dir., Vivere senza guerra. La pace nella ricerca universitaria, Milan, 1989, p. 201-218). Butturini multipliait ainsi les études sur la paix dans l'histoire du christianisme. La « paix juste » était l'aboutissement d'une réflexion lancée dans « La nonviolenza del Cristianesimo dei primi secoli. Antologia dei prosatori latini » (Turin, 1977) et poursuivie dans «La croce e lo scettro " (Florence, 1990), ouvrages d'où se dégageait une interprétation positive de la non-violence. Cette conception procédait des courants démocratiques du catholicisme et David Turoldo pouvait affirmer, dans sa préface du livre de Butturini sur le christianisme des premiers siècles, que la violence des sociétés modernes nous interdisait de parler de " guerre juste ", la seule justice étant désormais celle qu'il fallait rendre aux pauvres. Le titre «Le sceptre et la croix » avait été suggéré à l'auteur par Ernesto Balducci et Butturini rendait hommage à Primo Mazzolari (Emilio Butturini, Guerra e pace in don Primo Mazzolari, «Rivista lasalliana», $\mathrm{n}^{\circ}$ 2, 2009, p.199-205), mais le pédagogue de Vérone ne partageait pas le radicalisme de son préfacier. Sa conception de la paix s'inspirait davantage de «Pacem in terris» et des encycliques de Paul VI ou de Jean-Paul II. La culture laïque était d'ailleurs présente dans la réflexion de Butturini (Emilio Butturini, La pace giusta. Testimoni e maestri tra '800 e '900. Ruskin, Tolstoi, Gandhi, Montessori, Capitini, Milan, Vérone, 2007). La paix était inséparable d'une vision de la justice que Ruskin estimait bafouée par la misère et l'assujettissement de la personne. Maria Montessori dénonçait la guerre comme une maladie morale et sa sensibilité universelle l'orientait vers la formation d'un citoyen du monde. Quant à la laïcité de Capitini, elle faisait référence à la transcendance et restait ouverte aux instances religieuses de la vie. Les formes démocratiques de la communauté éducative étaient une initiation à la collaboration et à la libre critique. Elles devaient soustraire l'Histoire à une rhétorique exaltant la puissance de la guerre.

Tout en conservant un intérêt constant pour une approche concrète des problèmes éducatifs qui déchirent la famille de notre époque et compliquent les relations entre les générations (Emilio Butturini, Dialogo educativo con i giovani sui temi della verità, libertà e senso della vita, in: Mario Dal Corso, Educazione-formazione tra passato e futuro, Milan, 2006, p. 17-36), Butturini semblait s'inspirer d'un retour aux sources du christianisme pour évoluer vers un messianisme démocratique qui suscitait quelque perplexité. La pédagogie sociale de Lorenzo Milani, Balducci et Turoldo dont il se sentait proche oscillait en effet entre utopie et idéologie, ambiguïés et incertitudes, contradictions et superficialité. Le spiritualisme de la pensée laïque peut revêtir par ailleurs des formes très éloignées de l'ouverture d'esprit d'une laïcité positive et se confondre avec une religion des Droits de l'Homme dont Butturini avait condamné l'incapacité à tenir compte du fait religieux dans l'élaboration de la culture. Un mélange de théosophie et de culture orientale donne par exemple à la sensibilité religieuse de Montessori un caractère indéfini qui a suscité bien des interrogations. Le pédagogue de Vérone conservait pourtant un sens pratique qui lui évitait de tomber dans les excès où certains de ses inspirateurs s'étaient laissés choir. L'union de la notion de paix à celle de justice bannissait l'agression sans renoncer à une défense légitime et elle n'entendait pas apporter un supplément d'âme au pacifisme. Butturini puisait ce sens de la mesure dans un contact permanent avec les institutions religieuses de Vérone 
auprès desquelles il ne cessait de se ressourcer. Elles lui rappelaient constamment que l'éducation est la forme la plus élevée de la charité chrétienne. 